\title{
A hospital based study of assessment of missed opportunities of immunization in children aged less than two years
}

\begin{abstract}
Background: Immunization is the most cost effective ways to improve the health of a community and to avert vaccine-preventable diseases associated morbidity and mortality in children. The immunization status of young children of Western Rajasthan is not up to the mark despite of all the efforts and Missed opportunities of immunization is one of the important contributing factors. The present study is aimedto know prevalence of missed opportunities of immunization in children and the reasons for the same at our centre.
\end{abstract}

Methods:Immunization status was assessed via interview method using standard protocols recommended by the WHO, for a total of 600 children aged less than two years (200 indoor and 400 outdoor patients) picked up through a table of random numbers. The children who had missed an immunization opportunity were compared with those who had not, for sociodemographic variables and reasons for missed opportunity were determined.

Results: $48.5 \%$ of the children were immunized up to date, $33.82 \%$ were partially immunized not up to date and $15.17 \%$ were completely un-immunized. The status of $2.5 \%$ children could not be assessed and were excluded from the study. $45.64 \%$ of children had missed an opportunity for immunization. Illiteracy, higher birth order, rural residence, poor socioeconomic status emerged as the risk factors. Immunization status not assessed during health visit in $82.39 \%$, lack of knowledge of the parents regarding immunization schedule in $79.40 \%$ and false contraindications (mild acute illness in $21 \%$, current antimicrobial therapy in $8.22 \%$, convalescent phase of illness in $7.86 \%$, recent exposure to infectious disease in $5.24 \%$, fever precipitating seizures in $1.12 \%$, pre-maturity $0.74 \%$ in that order) were the main causes.

Conclusions:Immunization is an important but yet neglected part of child health visits. Each child's immunization status should be assessed at every health care visit to avoid missed opportunities

Keywords: missed opportunity, immunization, vaccine preventable diseases
Volume 6 Issue 5 - 2017

Suresh Kumar Verma,' Anita Yadav, ${ }^{2}$ Dabi DR ${ }^{3}$

'Professor of Pediatrics, Department of Pediatric Medicine, Dr.S. N. Medical College, India

${ }^{2}$ Consultant Pediatrician, Max Hospital, India

${ }^{3}$ Ex-Professor, Department of Pediatric Medicine, Dr. S. N.

Medical College, India

Correspondence: Suresh Kumar Verma, Department Of Pediatrics, Umaid Hospital Campus, India,

Email drsurehverma7l@gmail.com

Received: March 29, 2017| Published: May 04, 2017
Abbreviations: MOI, missed opportunities of immunization; WHO, world health organization; GOI, government of india; EPI, expanded program on immunization; OPD, out patient department; IUD, immunized up to date; PIN, partially immunized not up to date; IU, unimmunized; HHE, hypotensive hypo responsive episodes; IAP, indian academy of pediatrics; OR, odd ratio

\section{Introduction}

Immunizing children in the first years of life is one of the most effective ways to improve the health of a community and to decrease the costs associated with treating vaccine-preventable diseases. Even today the vaccine preventable diseases continue to take a high toll in the form of childhood mortality in spite of the fact that a readily available feasible and free of cost simple intervention of immunization exists in all the healthcare facilities across the country. In absolute figures India contributes to about $25 \%$ of the over 10.6 million under five deaths occurring worldwide every year. Most of these deaths are still attributable to just a handful of conditions are avoidable through existing interventions. ${ }^{1}$ According to GOI report, EPI has achieved very high coverage in India reported to have touched $90 \%$ of target children. (63\% fully immunized and $27 \%$ partially immunized) Non immunization was more frequent in the rural areas, schedule tribes and illiterate mothers of the state of Bihar, Rajasthan, UP and MP account for over two third of non immunized population. ${ }^{2}$ In the state of Rajasthan only $40 \%$ children are fully immunized, $33 \%$ partially immunized and $20 \%$ completely unimmunized. The situation is grimmer in our western desert part of the state; therefore it is essential to screen every child for immunization status at every visit to a clinic and should advise necessary immunization at every opportunity unless any true contraindications exist for the same. But many children visiting health facilities are partially immunized and the opportunity to immunization is missed even in teaching Hospitals. ${ }^{3}$ Missed opportunities are a hurdle for achieving full immunization coverage and by reducing it we can increase the immunization coverage in a cost effective manner in areas like western Rajasthan where the burden of disease targeted by EPI is still high and these missed opportunities of immunization may result in avoidable deaths and disability in children. This prompted us to conduct the study on assessment of missed opportunities of immunization in our area and the possible reason for the same.

\section{Materials and methods}

This cross sectional interview based case controlled descriptive study was carried out to assess the immunization status of children less than two years of age at the Department of Pediatrics, Umaid Hospital for women and children which is being a largest tertiary care center of Western Rajasthan attached to Dr. S.N. Medical College 
Jodhpur. This study was conducted over 600 children aged less than two years (200 indoor and 400 outdoor patients) who constituted our study material by using card and recall method to find out the prevalence of missed opportunities and delineate various factors contributing to the same. The present study used the standard protocols recommended by the WHO to assess missed opportunities of immunization in a tertiary teaching level hospital. The exact age of the child was confirmed via birth record, delivery notes immunization card or via recall of birth date, whenever possible. After the child had been attended by physician in OPD cases and given discharge from wards in case of admitted indoor cases, the accompanying attendant of these children were interviewed at the exit and all the personal social and demographic information were recorded over a pre designed Performa. The immunization status of every child was assed and labeled as Immunized up to date, partially immunized not up to date \& UN immunized as per following definitions. ${ }^{4}$

\section{Immunized up to date (IUD)}

A child who has received the entire vaccine dose as per his age recommended by the EPI.

\section{Partially Immunized Not up to date (PIN)}

A partially immunized child is one who has not yet received the entire vaccine dose as per his age recommended by the EPI.

\section{Un Immunized (IU)}

A child who has not yet received any vaccine dose as per his age recommended by the EPI.

To rule out any true contra indications for immunization, a complaint for which the child was brought to the hospital were taken in to consideration and if none were found then the child was consider as eligible for immunization. Persistent inconsolable cry for $>3 \mathrm{hrs}$ duration, hyperpyrexia (fever $>40.5 \mathrm{C}$ ), Hypo responsive episodes HHE (Collapse and shock like stage) within $48 \mathrm{hrs}$ of DTwP administration, seizures with or without within $72 \mathrm{hrs}$ of DPT administration were regarded as mere precautions because they do not cause permanent sequelae. If the similar adverse reaction occurred with the subsequent dose only then pertussis vaccine was taken as contraindicated as future administration as per IAP guideline on immunization in 2006.

The number of visits to health personnel made in past was noted. The knowledge of attendant was assessed by acquiring the detailed information regarding names of disease from which their children were protected from vaccination importance of immunization and the recommended timing of vaccine administration. A thorough search was made to elicit the reasons responsible for the child's incomplete immunization. In the end every missed opportunity was covered and the child immunized for all the vaccines for which he or she eligible with due emphasis on and motivation for immunization of other children in family and neighborhood.

\section{Results}

Out of all 600 children participated in this study, only $48.57 \%$ were immunized up to date, $33.82 \%$ were partially immunized not up to date and $15.17 \%$ were completely un-immunized. The status of $2.5 \%$ children could not be assessed and these children were excluded from the study (Figure-1). The $49 \%$ children who were not up to date with their immunization status, $45.64 \%$ were eligible for vaccination on the day of study but were not immunized thus had missed an opportunity for immunization. A male: female ratio of 1.93:1 was found significant in our study which indicates a persisting male bias in presentation to the health facility. Of the 267 children in MOI group, $64.79 \%$ were males and $35.20 \%$ were female whereas in non missed group these values were $66.98 \%$ and $33 \%$ respectively (Table -1 ). Place of delivery was significantly associated with MOI with less MOI in hospital delivered babies. In MOI group, only $52.80 \%$ children were delivered in hospital as compared to $72.95 \%$ for children who didn't miss an opportunity $(\mathrm{p}<0.001, \mathrm{OR}=4.34)$. Higher birth order was found to be significantly associated with MOI as revealed by figures of $31 \%, 36 \%, 18 \%, 6.7 \%, 6.7 \%$ children in MOI group having a birth order of one, two three, four, five or more respectively whereas in non missed group, the values were $40 \%, 30 \%, 21 \%, 4.7 \%, 3.77 \%$ $(\mathrm{OR}=3.99, \mathrm{p}=<0.1)$ (Table 1). However, religion did not emerge as a factor for $\mathrm{MOI}$ as we found that MOI occurred with similar frequency in both Hindus and Muslims ( $\mathrm{p}<0.8, \mathrm{OR}=4.03$ ). The Maternal literacy was found to be a key factor responsible for MOI .Our study revealed that MOI group babies had almost twice the number of illiterate mothers $(62.92 \%)$ as compared to control group $(32.2 \%)(\mathrm{p}<0.001)$ Also, the mothers in the latter group were higher in each of secondary, higher secondary, graduate and post graduate categories $(23 \%, 8.4 \%$, $8.4 \%, 1.2 \%$, respectively) when compared with former $(8.9 \%, 2.6 \%$, $1.4 \%, 0.37 \%$ ) (Figure 2). Similarly Education status of father also had a significant positive correlation with the child's immunization status $(p<0.001)$. Low socio-economic status was significantly related with MOI. ( $\mathrm{p}<0.001)$ We found that children in MOI group belonged seven fold less in number to socioeconomic scale one $(0.37 \%)$ and twice less to scale two $(4.49 \%)$ and three $(12.73 \%)$ as compared to children in non-missed group $(2.8 \%, 10 \%, 24 \%$ respectively), whereas the reverse was true for scale four and five $(78 \%, 3.74 \%$ respectively in MOI group and $60 \%, 1.57 \%$ in non missed group). Type of family (joint or nuclear), was found to have no bearing on MOI ( $>0.3$ ) in our study despite the common belief that elderly in joint families often refuse immunization. $47.94 \%$ of our MOI group kids belonged to rural and $52 \%$ to urban areas as compared to $26 \%$ and $73 \%$ in non-missed group $(\mathrm{p}<0.001)$ revealing rural residence to be associated with MOI. Of the 494 children who possessed an immunization card in our study, only $21 \%$ had brought it along with on this visit. Lesser $(12.5 \%)$ children in MOI group had brought their cards as compared to $25.78 \%$ in nonmissed group $(\mathrm{p}<0.001)$; bringing to light non availability of card to be a risk factor for MOI.

We found that $13.06 \%$ children in MOI group had local adverse reactions to previous vaccination as compared to the non-missed group where this figure was only $2.8 \%$, approximately four times less than the former $(\mathrm{p}<0.001, \mathrm{OR}=4.26)$. Fear of local adverse reaction to vaccination was cited as the cause of MOI in $21.72 \%$. We also found that children with missed opportunity had fewer number of previous health visits as compared to the non missed group ( $\mathrm{p}<0.001)$. We found that the most common reason for missed opportunities of immunization was failure on part of the treating doctor to assess the immunization status and subsequently advise age appropriate vaccination $(82.39 \%)($ Table-2). Only $13 \%$ parents would have refused vaccine administration today, had they been advised so by the treating physician. The next most common cause was ignorance of the immunization schedule by parents in $79.40 \%$ and false contraindications like mild acute illness in $21 \%$, current antimicrobial therapy in $8.22 \%$, convalescent phase of illness in $7.86 \%$, recent exposure to infectious disease in $5.24 \%$, fever precipitating seizures in $1.12 \%$, pre-maturity $0.74 \%$ in that order). Other causes being refusal by head of family in $4.8 \%$, distance of immunization centre from home in $4.11 \%$, unavailable vaccines either due to staff absenteeism $1.87 \%$ or immunization clinic closed $3.74 \%$, lack of knowledge (regarding age of administration in $3.74 \%$, newspaper generated fear in $1.49 \%$ ), tiny baby in $2.98 \%$, father not staying at home in 
$2.61 \%$, baby migration $1.49 \%$, attendant in hurry in $1.49 \%$, multiple injections in $1.12 \%$ cases (Table 2). A total 267 children who had missed an opportunity for immunization formed the case group and rest who were immunized up to date or partially immunized without any missed opportunity formed the control group for comparison of factors associated with MOI.

Table I Baseline Characteristics of the Study Sample

\begin{tabular}{|c|c|c|c|}
\hline Parameter Studied & Indoor ( $\mathrm{N}=197)$ & Outdoor $(\mathrm{N}=388)$ & Total $(\mathbf{N}=585)$ \\
\hline Sex & $150(76.14 \%)$ & $236(60.82 \%)$ & $386(65.98 \%)$ \\
\hline male & $47(23.85 \%)$ & $152(39.17 \%)$ & $199(34.01 \%)$ \\
\hline female & 3.91:1 & I.55:I & $1.93: 1$ \\
\hline \multicolumn{4}{|l|}{ M:F ratio } \\
\hline Place of delivery & $90(45.68 \%)$ & $122(3 \mid .44 \%) 266(68.55 \%)$ & $212(36.23 \%)$ \\
\hline Home & $107(54.13 \%)$ & & $373(63.76 \%)$ \\
\hline \multicolumn{4}{|l|}{ Hospital } \\
\hline Religion & $153(77.66 \%)$ & $153(77.66 \%)$ & $153(77.66 \%)$ \\
\hline Hindu & $44(22.33 \%)$ & $44(22.33 \%)$ & $44(22.33 \%)$ \\
\hline \multicolumn{4}{|l|}{ Muslim } \\
\hline Literacy Mother I & $101(51.26 \%)$ & $170(43.81 \%)$ & $27 I(46.32 \%)$ \\
\hline lliterate primary & $5 I(25.8 \%)$ & $101(22.03 \%)$ & $152(25.98 \%)$ \\
\hline Secondary & $56(25.88 \%)$ & $75(19.32 \%)$ & $99(16.92 \%)$ \\
\hline Hr. Sec. & $9(4.5 \%)$ & $18(4.63 \%)$ & $27(4.61 \%)$ \\
\hline Graduate & $12(6.09 \%)$ & $19(4.89 \%)$ & $31(5.2 \%)$ \\
\hline P.G. & $0(0 \%)$ & $25(1.28 \%)$ & $5(0.8 \%)$ \\
\hline Literacy Mother I & $49(24.87 \%) 5 \mathrm{I}(25.88 \%)$ & $83(21.39 \%)$ & $132(25.56 \%)$ \\
\hline lliterate primary & $56(28.42 \%)$ & $96(24.74 \%)$ & $147(25.12 \%)$ \\
\hline Secondary & $17(8.62 \%)$ & $119(30.67 \%)$ & I75(29.9|\%) \\
\hline Hr. Sec. & $24(12.18 \%)$ & $51(13.14 \%)$ & $68(11.62 \%)$ \\
\hline Graduate & $2(1.01 \%)$ & $84(21.64 \%)$ & $58(9.9 \%)$ \\
\hline P.G. & & $3(0.7 \%)$ & $3(0.5 \%)$ \\
\hline Socio economical Scale & $4(2.03 \%)$ & $6(1.54 \%)$ & $10(1.7 \%)$ \\
\hline I & $19(9.6 \%)$ & $26(6.7 \%)$ & $45(7.6 \%)$ \\
\hline 2 & $39(19.79 \%)$ & $74(19.07 \%)$ & $113(19.31 \%)$ \\
\hline 3 & $134(68.02 \%)$ & $268(69.07 \%)$ & $402(68.71 \%)$ \\
\hline 4 & $\mathrm{I}(0.50 \%)$ & $14(3.60 \%)$ & I5(8.7|\%) \\
\hline \multicolumn{4}{|l|}{5} \\
\hline Family Type & I I I(56.34\%) 86(43.65\%) & $242(62.37 \%) \quad \mid 46(37.62 \%)$ & $353(60.34 \%) 232(39.65 \%)$ \\
\hline \multicolumn{4}{|l|}{ Joint } \\
\hline \multicolumn{4}{|l|}{ Nuclear } \\
\hline Residence & $95(48.22 \%) \quad 102(51.77 \%)$ & $116(29.89 \%) \quad 272(70.01 \%)$ & $211(36.06 \%) 374(63.39 \%)$ \\
\hline \multicolumn{4}{|l|}{ Rural } \\
\hline Urban & & & \\
\hline
\end{tabular}

Table 2 Reasons for Missed Opportunity of Immunization

\begin{tabular}{llll}
\hline Reasons for Missed Opportunity & Indoor $(\mathbf{N}=\mathbf{I 0 2})$ & Outdoor $(\mathbf{N}=\mathbf{I 6 5})$ & Total (N=267) \\
\hline Immunization status not assessed & $85(83.33 \%)$ & $135(81.81 \%)$ & $220(82.34 \%)$ \\
Immunization not advised & $85(83.33 \%)$ & $135(83.33 \%)$ & $220(82.34 \%)$ \\
Ignored the immunization schedule & $67(65.68 \%)$ & $145(87.87 \%)$ & $212(79.40 \%)$ \\
Fear of local adverse reaction to vaccine & $12(11.26 \%)$ & $46(27.87 \%)$ & $58(21.72 \%)$ \\
Mid acute illness regardless of fever & $13(12.74 \%)$ & $34(20.60 \%)$ & $47(17.60 \%)$ \\
Current antimicrobial therapy & $8(7.80 \%)$ & $14(8.48 \%)$ & $22(8.22 \%)$ \\
Convalescents phase of illness & $12(11.76 \%)$ & $9(5.43 \%)$ & $21(7.86 \%)$ \\
Recent exposure of infectious disease & $5(4.90 \%)$ & $9(5.43 \%)$ & $14(5.24 \%)$ \\
Refusal by head of family & $3(2.90 \%)$ & $5(3.03 \%)$ & $13(4.8 \%)$ \\
Distance from immunization centre & $6(5.88 \%)$ & $5(3.03 \%)$ & $11(4.11 \%)$ \\
Immunization clinic closed & $10(9.8 \%)$ & $0(0 \%)$ & $10(3.74 \%)$ \\
Exact timing regarding vaccine not timing & $7(6.8 \%)$ & $3(1.81 \%)$ & $10(3.74 \%)$ \\
Child too small to be brought for vaccine & $3(2.90 \%)$ & $5(3.03 \%)$ & $8(2.98 \%)$ \\
Father not staying at home & $4(3.92 \%)$ & $3(1.81 \%)$ & $7(2.61 \%)$ \\
Lost immunization card & -- & $6(3.62 \%)$ & $6(2.24 \%)$ \\
Staff not regularly available at native centre & $2(1.96 \%)$ & $3(1.81 \%)$ & $5(1.87 \%)$ \\
Attendant in hurry & $1(0.98 \%)$ & $3(1.81 \%)$ & $4(1.49 \%)$ \\
Newspaper report two kid died post DPT & $1(0.98 \%)$ & $3(1.81 \%)$ & $4(1.49 \%)$ \\
Baby out of station & -- & $4(2.42 \%)$ & $4(1.49 \%)$ \\
Too many injections & $1(0.98 \%)$ & $2(1.21 \%)$ & $3(1.12 \%)$ \\
\hline
\end{tabular}




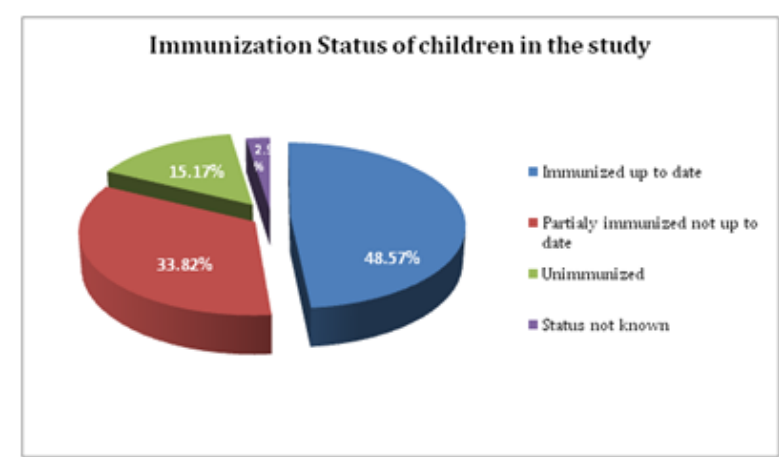

Figure I Pie diagram showing immunization status of children in study.

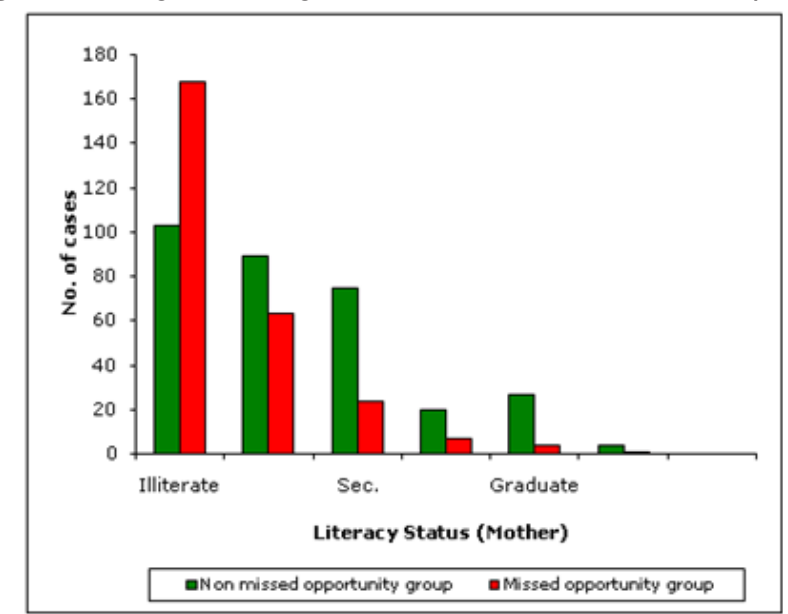

Figure 2 Educational level of mother and its relation to the immunization status of children.

\section{Discussion}

We found in the present study that the most common reason for missed opportunity of immunization was failure on the part of the health care personals to assess the immunization status among $82.39 \%$ of the children and subsequently advise them for age appropriate immunization. (Table 2) Each child's immunization status should be assessed at every health visit so as to avoid any missed an opportunity of immunization. Not offering measles vaccination at curative visit was the major reason of MOI in $86.9 \%$ children reported by Meera et al., ${ }^{5}$ Bell et al., ${ }^{6}$ Rey LC et al., ${ }^{7}$ Biswas et al., ${ }^{8}$ and Waltone et al., ${ }^{9}$ Have also attributed most of the missed opportunities to health care provider and delivery system of the studied hospital. As per study of Daniel et al., ${ }^{10} 90.5 \%$ of children had enough vaccination visit to complete the series, but had MOI due to failure to screen the status at each visit. The most disadvantaged, out of reach children presented to treating pediatrician only when acutely ill such children must be administered all the vaccine for which the child is eligible unless any true contraindication exists. Udovic et al., ${ }^{11}$ found that $80 \%$ would have accepted an immunization during the urgent care visit if advised by the physician. In our study parent's lack of awareness toward the immunization accounted for MOI in $79 \%$ cases. O G Seikh et al., ${ }^{12}$ also found ignorance in $42 \%$ urban and $49 \%$ of rural parents, whereas S Yadav et al. ${ }^{13}$ observed in $80 \%$, Malini et al. ${ }^{14}$ in $64 \%$, and Joseph et al. ${ }^{15}$ in $12 \%$ to be the cause of MOI. False contraindications regarding immunization substantially accounted for MOI, $1.12 \%$ children with simple febrile seizures feared fever after vaccination would precipitate seizures. Concurrent medication (8.22\%) recovering from illness $(7.86 \%)$ and recent exposure to an infectious disease were other false contraindications contributed for MOI. In the studies of
Brugha et al., ${ }^{16} \&$ Holt et al., ${ }^{17}$ also reported false contraindications to be an important factor for MOI. Acute illness is also found to be one of the reasons of MOI in $17.60 \%$ of the cases as there is general belief that children are not to be immunized during acute illness weather it is mid fever, diarrhea or URI. Similar results were reported in study of Joseph et al., ${ }^{15}$ Sebin et al., ${ }^{18}$ and Shahla et al. ${ }^{19}$ where they found child being unwell as a reason of MOI in $9 \%, 15.6 \%, \& 67 \%$ children respectively. Whereas Tarwa et al., ${ }^{20}$ found it to be the commonest reason for MOI. Since this acute illness takes maximum number of children to the physician, this false perception is to be taken care of by the treating physician in whom the patients confide. We also found too many injections, prematurity, refusal by head of the family, distance of immunization centre from home, tiny baby and father staying away from home were some of the other reason for MOI. Other reason like Unavailable vaccines lost of immunization card need to be taken care by health care centre to avoid MOI. In this part of the state migratory nature of population is an important hurdle in completion of vaccination, therefore it is important that immunization card of child should be updated and transferred along with so that the responsibility of further immunization is carried on to achieve completion.

\section{Conclusion}

The Medical community needs to intensify their efforts in assessing the immunization at every health visit so that no child needlessly suffers from vaccine preventable disease and barriers to immunization should be eliminated by improving the education status and awareness of parent. The suffering or death of even one child from a vaccine preventable disease in the present era is an unnecessary and avoidable tragedy.

\section{Limitation of the study}

Though we followed the standard protocols recommended by the WHO to assess MOI in a tertiary teaching level hospital \& the exact age of child was confirmed via birth record, delivery notes and immunization card whenever possible. We also used recall method to find out the birth date and the prevalence of missed opportunities and delineated various factors contributing for the same in the situation of unavailability of immunization card and records in the present study.

\section{Acknowledgements}

Manuscript writing and analysis done by Dr. Suresh Kumar Verma, Data collection done by Dr. Anita Yadav. Study designed, and research work guided by Dr. D.R. Dabi.

\section{Conflicts of interest}

Author declares there are no conflicts of interest.

\section{Funding}

None.

\section{References}

1. Indian Academy of Pediatrics.IAP Guide Book on Immunization, India. p.10-14.

2. Padam Singh, Yadav RJ. Immunization Status of children. Indian Pediatr . 2001;37(11):1194-1199.

3. Singh P, Yadav RJ. Immunization Status of children in BIMARU States. Indian J Pediatr. 2001;68(6):495-500.

4. Sato P. Protocol for Assessment of Missed Opportunities of Immunization. WHO/EPI/GEN. 2012; 88(6): 5-6. 
5. Meera Karunakaran, Pavitra Mohan. Measles Immunization coverage in Urban slums. Indian J Pediatr . 1999;66(4):505-509.

6. Bell TM, Servint JR.Missed Opportunities for Vaccination and the Delivery of Preventive care. Arch Pediatr Adolesc Med. 1996;150(8):947-949.

7. Rey LC. Missed Opportunities for immunization in a hospital in Paediatric Hospital in Fortaleza. J Pediatr (Rio J) . 1996;72(1): 9-13.

8. Biswas AB, Mitra NK, Nandy S, et al. Missed Opportunities of immunization in children. Indian J Public Health. 2000;44(1):23-27.

9. Walton S, Elliman D, Bedford H. Missed Opportunities to vaccinate children Admitted to A Pediatric Tertiary Hospital. Arch Dis Child. 2007;92(7):620-622.

10. Daniel D, Jiles RB, Klevens RM, et al. Under vaccinated AfricanAmerican Preschoolers: A Case of Missed Opportunities. Am J Prev Med . 2001;20(4):61-68.

11. Udovic SL, Lieu TA ,Black SB, et al. Parent Reports on willingness to Accept Childhood Immunization During Urgent Care Visit. Pediatrics. 1998;102(4): E47.

12. AI Seikh OG, Al-Samarrai JI, Al Sumaidaie MM, et al. Immunization coverage among Children Born Between 1989 and 1994 in Saladdine. Iraq Eastern Mediterranean Health Journal . 1999;5(5):933-940.

13. Yadav S, Mangal S, Padhiyar N, et al. Evaluation of Immunization coverage Urban Slum of Jamnagar city. Indian Journal of community Medicine . 2006;31(4):10-12.
14. Malini Kar, Reddaiah VP, Kant S. Primary Immunization Status of children in slum area of South New Delhi : The challenge of reaching Urban Poor. Indian J Community Med . 2001;26:151-154.

15. Joseph L Methew, Harsh Babbar, et al. Reasons for Non Immunization of children in an urban low income Group in North India. Trop Doct. 2002;32(3):135-138.

16. Sebin SS, Pomeranz AJ, Amateau MM. The Effect of Education, Feedback, and Provide Prompts on the Rate of Missed Vaccine Opportunities in a Community Health Centre. Clin Pediatr (Phila). 2003;42(2):147-145.

17. Brugha R. Missed Opportunities for Immunization at Curative and Preventive. Health Policy Plan. 1995;10(3):312-318.

18. Holt E, Guyer B, Hughart N, et al. The Contribution of Missed Opportunities to childhood Under immunization in Baltimore. Pediatrics. 1996;97(4):474-480.

19. Shahla Roodpeyma, Zinat kamali, Reza Babai,et al. ) Tajik Mother and Vaccination: Knowledge, Attitudes and Practice in Iran. Journal of Pediatrics Infectious Disease. 2007;2(1):29-34.

20. Tarwa, De Villiers. The Use of the Road to Health card In Monitoring Child health. SA Fam Pract. 2007;49(1) 